\title{
IoT Entity Management System (IoTEMS): Um Sistema de Gerenciamento de Entidades IoT
}

\author{
Gabriela Biondi $^{1}$, Fabrizio Borelli ${ }^{1}$, Dener Ottolini ${ }^{1}$, Carlos Kamienski $^{1}$ \\ ${ }^{1}$ Universidade Federal do ABC (UFABC)
}

\begin{abstract}
Smart Applications are implemented in distributed architectures and need to deal with a large volume of data, coming from different sources and with different formats. Proper handling of this data by the many hardware and software components that this distributed architecture may have depends on efficient access control so that data integrity and consistency is maintained. The purpose of this article is to present IoTEMS - IoT Entity Management System, an application responsible for manipulating FIWARE data, which operates in the NGSI-LD format, in entities that make up the Smart Applications scenarios. To validate the proposal, the SWAMP Platform was used, which operates in precision irrigation scenarios. The results showed that centralizing the manipulation of entities using IoTEMS ensured data integrity and consistency.
\end{abstract}

Resumo. As Aplicações Inteligentes são implementadas em arquiteturas distribuidas e precisam lidar com um grande volume de dados, vindos de fontes distintas e com formatos diferentes. A manipulação adequada desses dados por parte dos muitos componentes de hardware e software que essa arquitetura distribuida pode possuir, depende de um controle de acesso eficiente, para que a integridade e consistência dos dados seja mantida. O objetivo deste artigo é apresentar o IoTEMS - IoT Entity Management System, uma aplicação responsável pela manipulação dos dados do FIWARE, que opera no formato NGSI-LD, em entidades que compõem os cenários das Aplicações Inteligentes. Para validar a proposta foi utilizada a Plataforma SWAMP, que atua em cenários de irrigação de precisão. Os resultados mostraram que a centralização da manipulação das entidades usando o IoTEMS garantiu a integridade e consistência dos dados.

\section{Introdução}

As Aplicações Inteligentes podem ser divididas em quatro grandes pilares [Ahmed et al. 2016, Zyrianoff et al. 2020]: cidades [Zanella et al. 2014], campo [Tzounis et al. 2017], indústria [Sisinni et al. 2018] e saúde [Islam et al. 2015]. Em todos esses casos seu principal objetivo é utilizar os recursos de IoT para agregar valor a experiência de seus usuários através da coleta e distribuição de dados, além da otimização de processos e recursos.

A arquitetura das aplicações inteligentes é, na grande maioria dos casos, distribuída e essa característica traz diversos desafios para a área, como a manipulação de dados. Por possuírem cenários bastante distintos, o conjunto de entidades virtuais que armazena esses dados precisa ser controlado de maneira centralizada, dentro da arquitetura distribuída. Os desenvolvedores, integradores e administradores de sistemas IoT necessitam de ferramentas para facilitar a manipulação das entidades virtuais que representam os 
diversos tipos de dispositivos físicos (como sensores e atuadores), equipamentos (como semáforos, robôs e sistemas de irrigação), ambientes (como fábricas, ruas, e fazendas) e dados calculados (como os resultados dos modelos que geram a tomada de decisão).

Com frequência as plataformas de IoT, principalmente as de código aberto como o FIWARE [Rodriguez et al. 2018], não oferecem interfaces amigáveis para os usuários realizarem operações CRUD (Create, Read, Update, Delete), assim como não oferecem uma visão geral do conjunto de entidades e seus relacionamentos. Plataformas específicas ainda pecam pela carência de interfaces necessárias para compreender ações importantes tomadas pelo sistema. Por exemplo, a plataforma FIWARE é caracterizada pelo broker de contexto Orion baseado no modelo publish/subscribe. Quando o conteúdo de uma entidade é atualizado, todos os serviços inscritos nela são notificados. No entanto, não há uma maneira simples de identificar quais serviços estão subscritos a quais entidades, tornando complexa a tarefa de gerenciar uma aplicação de IoT.

Esse artigo apresenta a ferramenta IoTEMS (IoT Entity Management System), uma aplicação que centraliza a responsabilidade de manipular todas as entidades de uma Aplicação Inteligente que funciona em uma arquitetura distribuída baseada em FIWARE. Ela foi construída durante a experiência dos autores no projeto $\mathrm{SWAMP}^{1}$ utilizando o FIWARE para construir uma plataforma para irrigação inteligente na agricultura. O IoTEMS é composto por cinco componentes principais, necessários para agilizar a realização de tarefas que exigiriam esforço manual: (1) IoTEE: IoT Entity Editor; (2) IoTEG: IoT Entity Grapher; (3) IoTEC: IoT Entity Configurator; (4) IoTEH: IoT Entity Historian e (5) IoTES: IoT Entity Suscriber. Para validar esta abordagem exploramos um cenário do campo com a plataforma SWAMP para irrigação de precisão na agricultura. Esta plataforma possui um conjunto vasto de hardwares e softwares que compõem a sua arquitetura distribuída. Todos esses componentes manipulam um conjunto de entidades, típicas de cenários de agricultura, com auxílio do IoTEMS.

Na sequência desse artigo, a seção 2 discorre sobre aplicações inteligentes baseadas em IoT, a seção 3 apresenta arquiteturas e plataformas de IoT, a seção 4 apresenta o IoTEMS e sua demonstração. Finalmente, a seção 5 apresenta conclusões relevantes.

\section{Aplicações Inteligentes baseadas em IoT}

As Aplicações Inteligentes estão presentes em todo o mundo e podem se dividir em quatro grandes pilares [Pallavi et al. 2017]: (a) o pilar com aplicações voltadas para as cidades; (b) um pilar com aplicações voltadas para o campo; (c) um pilar com aplicações voltadas para a indústria; e (d) um pilar com aplicações voltadas para a saúde.

Nas cidades, podemos encontrar exemplos de aplicações que se preocupam com os principais desafios encontrados em grandes metrópoles como, por exemplo, a mobilidade urbana. Há também aplicações voltadas para iluminação de vias públicas, do ponto de vista dos veículos e dos pedestres, e também há aplicações voltadas para iluminação de estradas. Todos esses projetos utilizam complexos esquemas de sensores para identificar quais áreas precisam ficar mais, ou menos, iluminadas, garantindo assim a segurança de todos e ainda assim economizando energia.

No campo, os desafios estão relacionados à otimização: a intenção é maximizar

\footnotetext{
${ }^{1}$ http://swamp-project.org
} 
os recursos produzidos, sejam eles animais para consumo ou plantações diversas, e minimizar o desperdício de insumos necessários nesse processo, como água nas plantações ou alimentos para os animais. Do ponto de vista das plantações, a irrigação de precisão está entre as aplicações mais procuradas, pois com seu uso o consumo de água é reduzido, afinal a plantação só é irrigada quando e quanto for necessário. Além da economia da água, há também uma melhoria observada na qualidade da plantação, pois a água é distribuída na medida certa, considerando o clima, o solo e as necessidades da planta.

$\mathrm{Na}$ indústria os desafios estão relacionados a automatização e otimização de processos, desde a aquisição de matérias primas, passando pelos processos de fabricação, venda e, por fim, chegando aos controles gerenciais de todas as etapas. As aplicações são muito variadas e adequadas a cada necessidade da fábrica em específico, mas esse conjunto vem sendo chamado de aplicações para Indústria 4.0.

$\mathrm{Na}$ saúde os desafios estão relacionados à diagnóstico precoce de doenças, monitoração de pacientes e controle de doenças infectocontagiosas, como é o caso da pandemia da COVID-19. Podemos encontrar com bastante facilidade aplicações que tem por objetivo monitorar sinais vitais básicos de uma pessoa, como batimentos cardíacos, pressão arterial, oxigenação e deslocamento ao longo do dia, a partir do uso de algum equipamento que possui sensores com estes fins.

\section{Arquiteturas e Plataformas de Aplicações de IoT}

As arquiteturas em camadas são frequentemente empregadas na construção de sistemas distribuídos, assim como em aplicações inteligentes, pois facilitam a compreensão de diferentes níveis de abstração de seus componentes [Zyrianoff et al. 2020]. A partir de uma arquitetura de IoT, arquiteturas de software para diversas aplicações podem ser derivadas, as quais definem a estrutura de alto nível de um sistema, compreendendo os componentes de software e suas relações [Bachmann et al. 2010]. A ferramenta apresentada neste artigo está preocupada em como criamos e configuramos diferentes entidades em arquiteturas para IoT. Hoje em dia a maior parte dos esforços nesse sentido estão concentrados na melhoria do gerenciamento dos dados distribuídos, a fim de disponibilizar os dados prontamente para o processamento de diferentes aplicações [Khan et al. 2012]. A proposta deste artigo não foca apenas nos dados produzidos pelos sensores, mas sim no gerenciamento de cada componente, permitindo ainda a visualização de sua relação com demais componentes, séries históricas, e propagação das alterações deste componente, fator comum em ambientes distribuídos.

Devido à natureza distribuída inerente dos aplicativos IoT, é imperativa a necessidade de implementar infraestruturas altamente distribuídas que vão desde as coisas inteligentes, passando por diferentes estágios de processamento/armazenamento/transmissão, até a nuvem e, posteriormente, ao terminal do usuário. Em outras palavras, defendemos que as arquiteturas de IoT devem ter um papel ativo na implantação de novas aplicações.

Uma plataforma IoT implementa uma arquitetura IoT fornecendo uma variedade de blocos de construção para facilitar o desenvolvimento de um aplicativo inteligente IoT, como conectividade de dispositivo, gerenciamento de dispositivo, gerenciamento de dados, análise de dados, segurança e visualização. Uma plataforma IoT reúne dados de dispositivos IoT e permite o desenvolvimento de aplicações inteligentes que controlam, monitoram e gerenciam esses dispositivos. Muitas vezes, é composta de vários com- 
ponentes de middleware, cada um focando em um recurso específico em uma camada específica para fornecer uma plataforma ponta a ponta envolvendo geração, transmissão, armazenamento e processamento de dados [Rodriguez et al. 2018].

Os profissionais podem contar com diferentes plataformas de IoT disponíveis, tanto de código aberto quanto de soluções comerciais. Essas plataformas podem usar vários protocolos e padrões ou mesmo soluções proprietárias. Essa abundância de plataformas leva a problemas de interoperabilidade, uma vez que os desenvolvedores devem adaptar suas aplicações a cada plataforma, usando API, modelos de informação e protocolos específicos. Portanto, as interfaces padrão são necessárias para atender a esse problema de interoperabilidade entre aplicações IoT e plataformas IoT heterogêneas. Além disso, os requisitos de segurança devem ser atendidos, tais como autenticação, autorização e controle de acesso e comunicação segura [da Cruz et al. 2018].

A Plataforma FIWARE tem atraído a atenção geral por ser uma solução de código aberto composta por uma série de componentes de software chamados Generic Enablers (GE) que executam funções em aplicações inteligentes [Rodriguez et al. 2018]. Os GEs podem ser usados para construir diferentes aplicações que trocam informações por meio de uma API REST seguindo o padrão NGSI baseado em JSON. O aspecto central do modelo de informações do FIWARE NGSI Context Management é o conceito de entidades e seus atributos. Devido aos avanços recentes em Linked Data (LD), tem havido um esforço contínuo para evoluir a API NGSI para NGSI-LD, que usa JSON-LD para serializar dados semânticos RDF (Resource Description Framework). Tanto o NGSI quanto o NGSI-LD são atualmente suportados pelo FIWARE, embora a conformidade total do FIWARE com o NGSI-LD ainda esteja em andamento. Alguns FIWARE GEs são considerados facilitadores-chave, como:

- Orion: um broker de contexto publish/subscribe, considerado o coração do FIWARE. O Orion armazena apenas a versão mais recente dos atributos da entidade e precisa trabalhar em conjunto com outros aplicativos para manter os dados históricos.

- IoT Agent: Mapeia dados vindos de sensores e indo para atuadores para o modelo de informações FIWARE NGSI para serem armazenados no Orion e posteriormente processados por outros GEs ou aplicativos externos.

- Quantum Leap: Preserva os dados históricos da entidade do Orion NGSI como séries temporais.

\section{IoTEMS: IoT Entity Management System}

O IoTEMS foi construído para facilitar a manipulação de entidades em uma solução de IoT baseada em FIWARE, permitindo assim que diferentes aplicações recebam o reflexo desta manipulação de maneira sincronizada, mantendo assim a consistência dos dados. A principal vantagem de se adotar essa ferramenta no desenvolvimento de uma aplicação para IoT é que a manipulação de entidades pode ser feita por pessoas com pouco conhecimento técnico, já que a interface gráfica abstrai boa parte da complexidade das operações. O IoTEMS contém cinco componentes principais: (1) IoTEE: IoT Entity Editor; (2) IoTEG: IoT Entity Grapher; (3) IoTEC: IoT Entity Configurator; (4) IoTEH: IoT Entity Historian e (5) IoTES: IoT Entity Suscriber. Cada um deles é responsável por um conjunto de funções que atuam sobre as entidades de uma aplicação inteligente. 


\subsection{IoTEE: IoT Entity Editor}

O IoTEE permite ações CRUD (Create, Read, Update, Delete) nas entidades usadas pela aplicação IoT. Ele armazena as entidades no FIWARE Orion que por sua vez as armazena no MongoDB, permitindo que um sistema IoT trate de dados provenientes de sensores, e dados computados pelos modelos relacionados às aplicações, para alterar o estado dos atuadores (por exemplo, ligar ou desligar a irrigação). O IoTEE fornece uma interface gráfica amigável que elimina a necessidade dos usuários manipularem APIs complexas usando programas.

A Figura 1 mostra a interface principal do IoTEE com a lista das entidades de um modelo de informações de irrigação inteligente [Kamienski et al. 2019], enquanto que a Figura 2 mostra a representação de uma entidade quando o usuário escolhe a opção de Edit/View. Essas opções permitem que o usuário edite as entidades através de uma interface gráfica, assim como permitem a edição direta do JSON no formato NGSI.

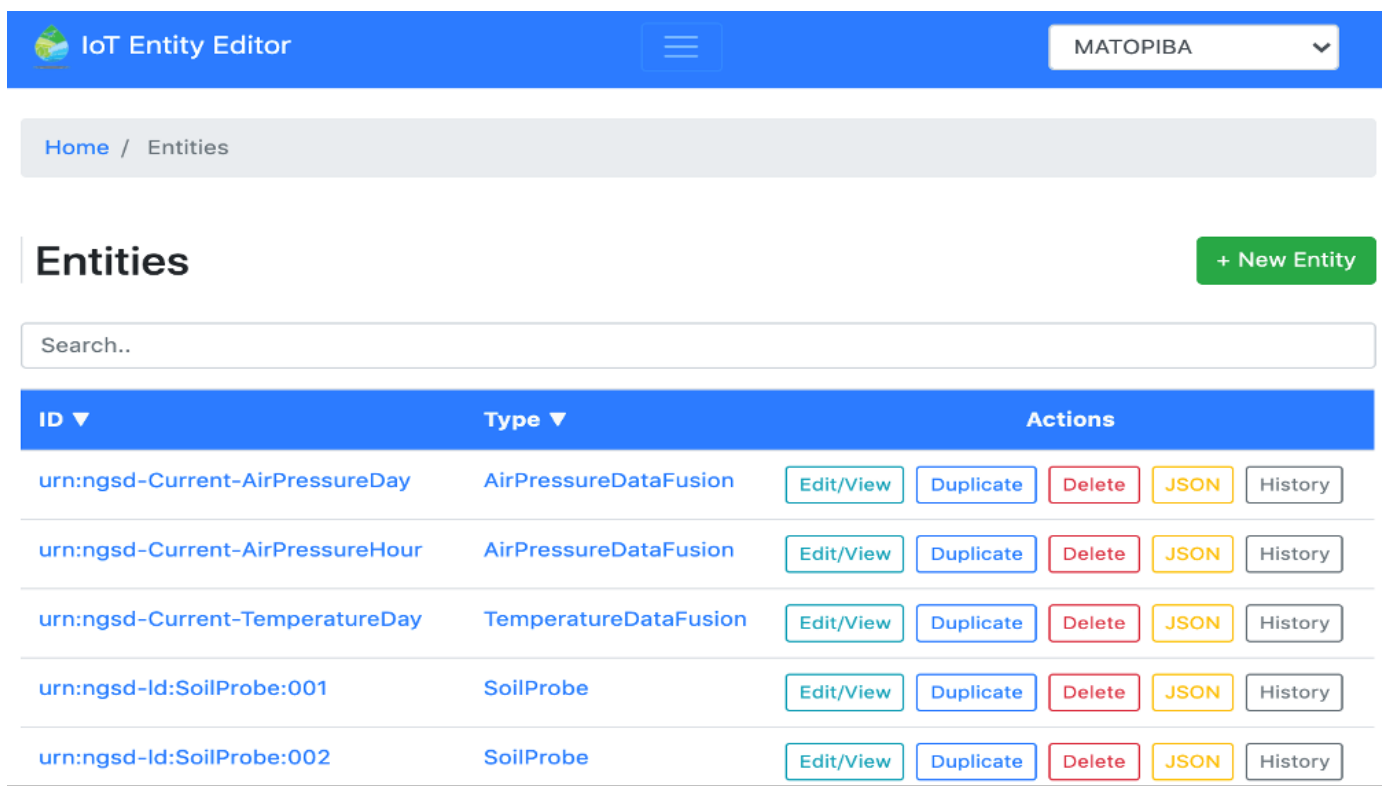

Figura 1. IoTEE: IoT Entity Editor - Operações CRUD

\subsection{IoTEG: IoT Entity Grapher}

Todas as entidades manipuladas pelo IoTEMS são escritas em JSON e armazenadas no Orion. Muitas vezes essas entidades apresentam algum tipo de relacionamento, como por exemplo a zona de manejo e a plantação. Neste caso, a visualização deste relacionamento através de arquivos escritos em JSON é dificultada. A solução proposta no IoTEG é mostrar cada entidade como um vértice e seus relacionamentos como arestas em um grafo. Como mostrado na Figura 3.

\subsection{IoTEC: IoT Entity Configurator}

Quando um novo dispositivo é adicionado ao cenário manipulado pelo IoTEMS, existe a preocupação de configurar esse novo dispositivo em todos os componentes de software que devem manipulá-lo. O IoTEC automatiza o fluxo que vai fazer essas configurações em cada componente de software. O IoTEC é um RPA (Robotic Process Automation) 


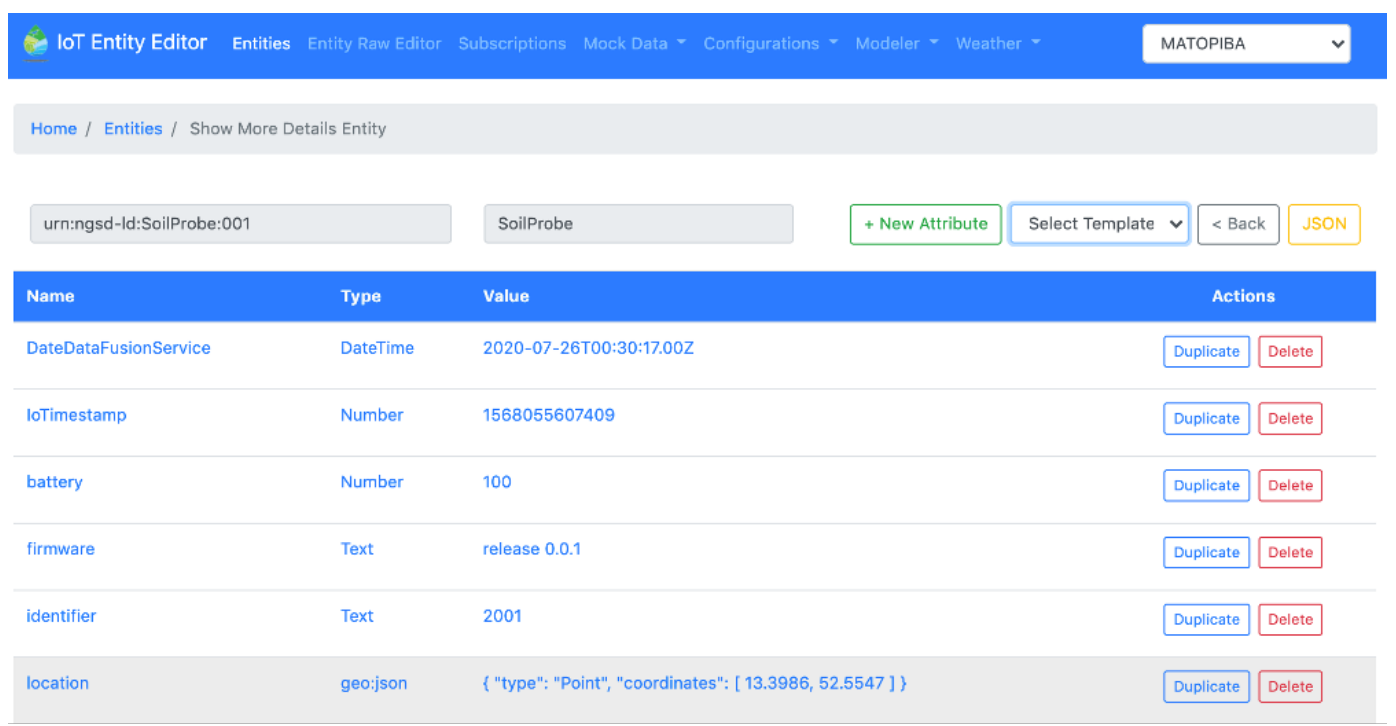

Figura 2. IOTEE: loT Entity Editor - Visualizar e editor entidades

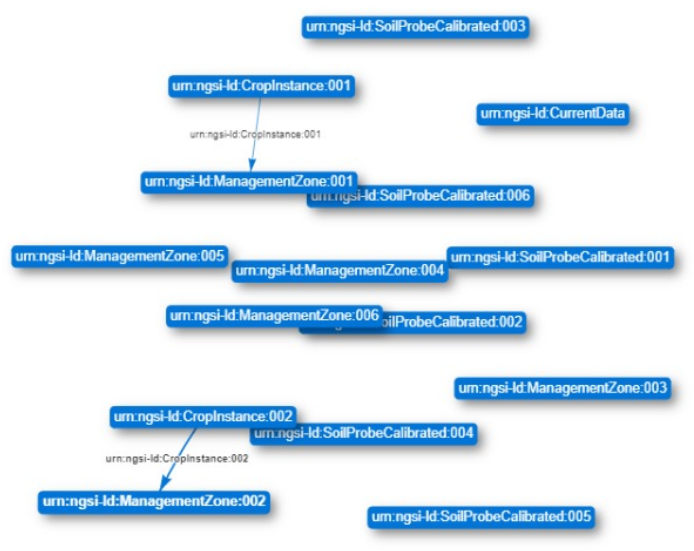

Figura 3. IoTEG: IoT Entity Grapher

composto de um conjunto de scripts para detectar a criação de novos dispositivos no Orion e automatizar a criação de representações dos dispositivos no IoT Agent e o ChirpStack LoraWAN Server.

\subsection{IoTEH: IoT Entity Historian}

Uma vez criadas e configuradas, as entidades começam a armazenar seus valores que podem ser visualizados em um formato de séries temporais. Essa visualização permite que os valores sejam plotados em função do tempo e, assim, a sua variação ao longo do tempo pode ser percebida, como pode ser observado na Figura 4.

\subsection{IoTES: IoT Entity Suscriber}

Outra preocupação, após a criação e configuração de uma nova entidade, é o gerenciamento de acessos, em outras palavras, quais componentes de software terão permissão para manipular os valores dessa entidade, seja lendo e/ou escrevendo valores nela. $\mathrm{O}$ IoTES é responsável por controlar as subscrições nas entidades do Orion, conforme mostrado na Figura 5. 


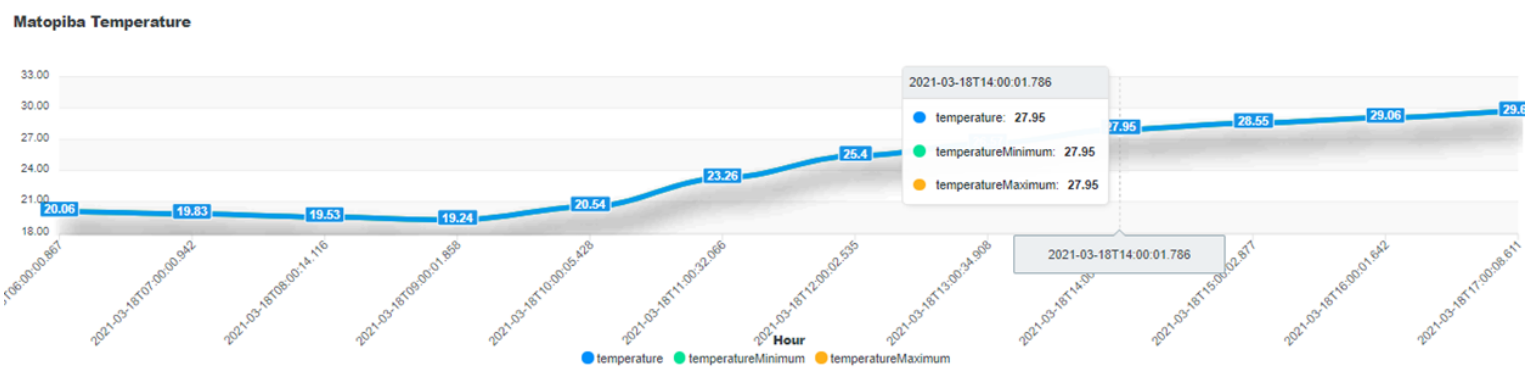

Figura 4. IoTEH: IoT Entity Historian

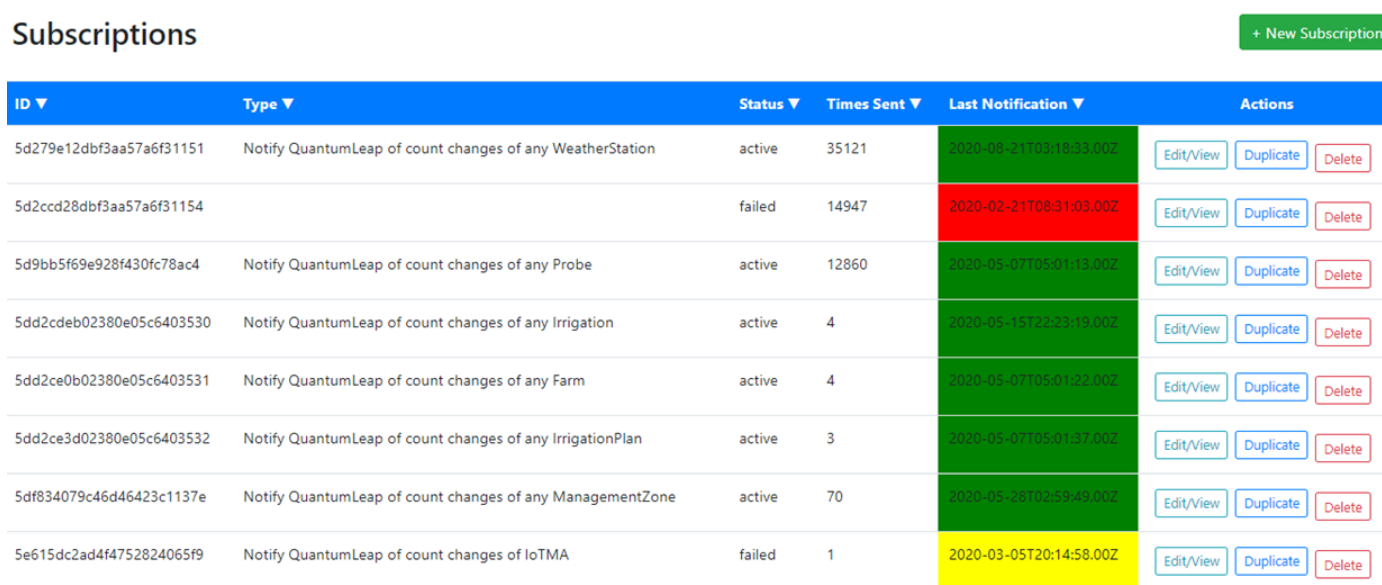

Figura 5. IoTES: loT Entity Subscriber

\subsection{Demonstração}

A demonstração da ferramenta será feita utilizando um cenário de irrigação inteligente do Projeto SWAMP, baseado em FIWARE. Com arquitetura de hardwares e softwares construídas, precisamos criar as entidades que definirão o cenário em questão, como a zona de manejo, talhão, espécie de planta, características do solo e irrigação. Essas entidades precisam ser criadas e configuradas, e precisam estar disponíveis para todas as aplicações de maneira consistente. A demonstração seguirá o tutorial ${ }^{2}$, de acordo com o vídeo ${ }^{3}$. A ferramenta está disponível online ${ }^{4}$, mas também pode ser encontrada no repositório com todos os códigos do projeto ${ }^{5}$. É necessário acesso ao FIWARE para instação.

\section{Conclusão}

As aplicações construídas em Arquiteturas de IoT possuem muitas camadas de hardware e software, e as entidades criadas para suportar os cenários manipulados pela aplicação precisam estar consistentes em todas as camadas. O IoTEMS foi construído para facilitar a manipulação de entidades em uma arquitetura de IoT baseada em FIWARE, permitindo assim que diferentes aplicações recebam o reflexo desta manipulação de maneira sincronizada. A principal vantagem de se adotar essa ferramenta no desenvolvimento de

\footnotetext{
${ }^{2}$ https://drive.google.com/file/d/15DH4rMFwCR5TCLuwNOCEj0Lscg-bGiAy

${ }^{3}$ https://www.youtube.com/watch?v=6Jdq8LkHu90

${ }^{4}$ iotee.swamp-project.org

${ }^{5}$ https://git.rnp.br/swamp-essentials/management/swamp-iot-ee
} 
uma aplicação para IoT é que a manipulação de entidades pode ser feita por pessoas com pouco conhecimento técnico, já que a interface gráfica abstrai boa parte da complexidade das operações. O uso da ferramenta foi eficiente durante o projeto SWAMP.

\section{Referências}

Ahmed, E., Yaqoob, I., Gani, A., Imran, M., and Guizani, M. (2016). Internet-of-thingsbased smart environments: state of the art, taxonomy, and open research challenges. IEEE Wireless Communications, 23(5):10-16.

Bachmann, F., Bass, L., Clements, P., Garlan, D., Ivers, J., Little, M., Merson, P., Nord, R., and Stafford, J. (2010). Documenting Software Architectures: Views and Beyond. Addison-Wesley Professional, second edition.

da Cruz, M. A., Rodrigues, J. J., Sangaiah, A. K., Al-Muhtadi, J., and Korotaev, V. (2018). Performance evaluation of iot middleware. Journal of Network and Computer Applications, 109:53-65.

Islam, S. R., Kwak, D., Kabir, M. H., Hossain, M., and Kwak, K.-S. (2015). The internet of things for health care: a comprehensive survey. IEEE access, 3:678-708.

Kamienski, C., Soininen, J.-P., Taumberger, M., Dantas, R., Toscano, A., Salmon Cinotti, T., Filev Maia, R., and Torre Neto, A. (2019). Smart water management platform: Iot-based precision irrigation for agriculture. Sensors, 19(2):276.

Khan, R., Khan, S. U., Zaheer, R., and Khan, S. (2012). Future internet: the internet of things architecture, possible applications and key challenges. In 2012 10th international conference on frontiers of information technology, pages 257-260. IEEE.

Pallavi, S., Mallapur, J. D., and Bendigeri, K. Y. (2017). Remote sensing and controlling of greenhouse agriculture parameters based on iot. In 2017 International Conference on Big Data, IoT and Data Science (BID), pages 44-48. IEEE.

Rodriguez, M. A., Cuenca, L., and Ortiz, A. (2018). Fiware open source standard platform in smart farming-a review. In Working Conference on Virtual Enterprises, pages 581589. Springer.

Sisinni, E., Saifullah, A., Han, S., Jennehag, U., and Gidlund, M. (2018). Industrial internet of things: Challenges, opportunities, and directions. IEEE Transactions on Industrial Informatics, 14(11):4724-4734.

Tzounis, A., Katsoulas, N., Bartzanas, T., and Kittas, C. (2017). Internet of things in agriculture, recent advances and future challenges. Biosystems Engineering, 164:3148.

Zanella, A., Bui, N., Castellani, A., Vangelista, L., and Zorzi, M. (2014). Internet of things for smart cities. IEEE Internet of Things journal, 1(1):22-32.

Zyrianoff, I., Heideker, A., Silva, D., Kleinschmidt, J., Soininen, J.-P., Salmon Cinotti, T., and Kamienski, C. (2020). Architecting and deploying iot smart applications: A performance-oriented approach. Sensors, 20(1):84. 